\title{
Das Doppelgesicht der Scham
}

Uwe Gerber

Religion vollzieht sich als Kampf um Macht. Religion wird überliefert und ist stets neu auszuhandeln.Viele Menschen mussten und müssen Religion hinnehmen um ihres eigenen Überlebens willen. Religion wird ausgefochten zwischen Göttinnen, zwischen Göttern, zwischen Göttinnen und Göttern, zwischen Gottheiten und Menschen, zwischen Menschen, zwischen Männern und Frauen. Macht-, Gender- und Schuldfragen wurden und werden grundlegend bestimmt als religiöse Dynamiken. Und dies geht nie ohne Pannen, Peinlichkeiten und Beschämungen $\mathrm{ab}$. Religion spielt sich im Spannungsfeld von Herrschaft und Beherrschtwerden, von Neugier und Scham, von Gebieten und Bestrafen, von Zeugen und Ermorden ab, wie Schöpfungsmythen, Mythen vom Schuldig- und Bestraftwerden der ersten Menschen, vom Brudermord zwischen Kain und Abel erzählen.

\section{Über Gott-Mensch-Beziehungen in den Schöpfungs- Mythologien und in dem Mythos vom Sündenfall}

Unter der Überschrift Mühsal der Besten lässt Brechts Herr Keuner uns einen besonderen Einblick in ein Handeln geben, das auf eine peinliche Art von Schaffen, Gestalten und Schöpfung hinweisen kann, und das uns, wenn wir dieses Dictum auf den jüdisch-christlichen Gott anwenden, ein mindestens ungewohntes Gottes-Bild zeigt: »Woran arbeiten Sie? wurde Herr K. gefragt. Herr K. antwortete: IIch habe viel Mühe, ich bereite meinen nächsten Irrtum vor« - also nicht: ॥Und Gott sah alles an, was er gemacht hatte, und siehe, es war sehr gut." (1. Mose 1,31) Wenn Schöpfung solche Prozesse meint, in denen Ordnung aus Chaos wird (wie es z.B. die priesterliche Schöpfungserzählung in 1. Mose 1 unter Rückgriff auf babylonische Götterkampf- und siebentägige Weltordnungsmythologie erzählend bekennt) und umgekehrt aus Ordnung Unordnung und Untergang werden (wie es der Natur-Mythos der Sintflut als Zerstörung der ersten Schöpfung spannend zu berichten weiß, und wie etliche apokalyptische Ausmalungen der dramatischen Umwandlung der Schöpfungswelt durch den höchsten Gott zu wissen vorgeben - zum Ansporn im endzeitlichen Endspurt auf die jenseitige Erlösungswelt), dann gibt es Interessantes zu erzählen; und es gilt, anders 
nachzudenken über die früh mit Irrtümern durchsetzte und als peinlich empfundene(n) Schöpfungsveranstaltung(en) des höchsten Gottes. Im Folgenden konzentriere ich mich auf die gescheiterte (?) Schöpfungsperformance mit der ersten Adam-Frau Lilith und deren metaphysisch revoltierenden Auszug aus Jahwes (und Adams) Machtbereich, dann auf die nachrückende Eva-Frau Adams und deren gemeinsamen Test mit Gott gemäß dem Mythos vom Sündenfall. Diese Mythen stehen zwar am Anfang der jüdisch-christlichen Bibel, aber sie sind überlieferungs- und kompositionsgeschichtlich relativ spät als Auftakt der Heils- und Unheilsgeschichte Jahwes/ Elohims mit seinem auserwählten Volk als Abschluss ’nach vorne< an den Anfang der Bibel gesetzt worden.

Vielleicht hat Emile M. Cioran diesen (in der Bibel verdrängten) Lilith-Komplex gemeint: "Indem man dem offiziellen Gott die Funktion des Vaters, des Schöpfers, des Managers aufdrängte, setzte man ihn Angriffen aus, denen er erliegen mußte. « Dass mit dem Erschaffungsprojekt in seinen Varianten mindestens des jahwistischen >Paradies-Gartens Eden und der priesterschriftlichen Erschaffung in sieben Tagen etwas nicht stimmt, etwas schief gelaufen sein könnte, das man "wie eine Missgeburt« zu erfahren bekommt, die sich nicht blicken lassen darf und im Verborgenen bleiben sollte, schimmert als Lilith-Mythos durch und haben Gnostiker und Manichäer als Peinlichkeit des israelitisch-jüdisch-christlichen Schöpfungsglaubens bearbeitet und manifest gemacht mit ihrer dualistischen Zuschreibung an einen guten Gott ohne Schöpfung und einen bösen Gott mit defizienter Schöpfung. Sie wollten sich nicht mehr als für den Erdenmenschen notwendig auf das sarkisch-fleischliche Erdenleben einlassen und hielten dem ewigen Geistfunken und seinen Ablegern in Gestalt geschöpflicher Menschen eine meta-physische, paradiesische Selbstfindungsregion ohne fleischliche Anfechtungen, ohne lange Diskurse über gut und böse und ohne Schämen offen. Der Kampf ums Menschsein begann mit der Erschaffung des Menschen als eines Paares, dem der Anreiz der Symmetrie in der Differenz als unstillbares Begehren mit jeweils fruchtbarem oder tödlichem Ausgang eingeschrieben bleibt. Diese Differenz im Kulturellen und Sexuellen, im Gestalten und Erzeugen macht kontingentes Menschsein ebenso aus wie die Sehnsucht der Menschen nach der Aufhebung dieser Differenz(en) in ihnen selbst und in ihren Beziehungen, nach Identität, nach Einssein ohne Verantwortung und ohne Schuldigwerden und Vergeben. Welche Macht hat der Mensch, um die eingeschriebene Differenz aufzuheben durch eine religiös motivierte Rückkehr in sein Herkunftsparadies spätestens am Ende der 
göttlichen Heilsgeschichte oder die Differenz zum Verschwinden zu bringen mit den neuzeitlichen Mitteln einer technokratischen und ökonomistischen horizontalen Heilsgeschichte oder die Differenz in ihrer Ambivalenz zu leben?

\section{Machtkampf Nr. 1: Schwierig- und Peinlichkeiten bei der dreifachen Erschaffung des Menschen}

Unternehmen wir also einen weiteren Versuch, so etwas wie Szenarien in dem Schöpfungsdispositiv zu rekonstruieren bis hin zu dem bekannten vaticinium ex eventu des >Sündenfalls` (1. Mose 3), dass das erste Menschenpaar vor die Wahl gestellt war: entweder wie der Schöpfer-Gott sichere Kenntnis über gut und böse zu haben in der Art eines un-menschlichen, weil gottanalogen Allmachtstatus ohne Sterblichkeit (dogmatisch als Spekulationsobjekt eines supralapsarischen status integritatis vorgestellt) oder ein risikoreiches, weil diesseits von gut und böse sich abspielendes, freud- wie leidvolles, sterbliches Leben als Makel zu führen. Ersteres ist als religiöses Heilsziel bis heute wach geblieben, und es ist neuerdings vor allem als gentechnisch induziertes Wunschobjekt virulent, letzteres stellt die blanke Alltagsrealität dar.

Im Folgenden wird (1.) eine kurze Rezeptionsgeschichte der Lilith-Mythologie vorgestellt, um dann (2.-4.) die drei Schöpfungsvarianten der Erschaffung des Menschen vorzustellen.

(1.) Die Rezeption der verdrängten vielgestaltigen Lilith. Die hier involvierte, aber beinahe vergessene und oft verdrängte erste Frau Adams, die Figur der Lilith, trat immer wieder ins Rampenlicht als Muster menschlicher Beziehungstragödien: als Leben spendende Göttin und als verführende, sogar Kinder tötende Dämonin. Manche sehen in ihr die weibliche Seite Gottes (als Vorgängerin der Sophia) bis hin zur Personifizierung als Verführerin der Männer, so auch der Frauenliebhaber Goethe im >Faust: » Wer ist denn das? «, fragt Faust vielleicht neugierig oder vielleicht ängstlich, und Mephisto gibt ihm zur Antwort: »Betrachte sie genau! Lilith ist das." Und Faust halb unwissend und halb ungläubig, vielleicht den Vorsichtigen oder eher den Barschen gebend: »Wer?«, und darauf Mephisto, vielleicht warnend oder eher einladend: »Adams erste Frau. Nimm dich in Acht vor ihren schönen Haaren, vor diesem Schmuck, mit dem sie einzig prangt. Wenn sie damit den jungen Mann erlangt, so läßt sie ihn so bald nicht wieder fahren." (Faust, 1. Teil, Walpurgisnacht)

Lilith ist ein literarisches Thema geblieben, angefangen von sumerischen Texten über israelitische (Bibel-)Texte, den Talmud, den 
Sohar (das Heilige Buch der Kabbala), den 19. Gesang in Dantes Göttliche Komödie bis zu dem Gedichtzyklus Jakob und Lilith von Wolf Peter Schnetz (2002), den beiden Beiträgen Lilith and Eve von Jakov Lind (1976) und Das Kommen Liliths von Judith Plaskow (1982), Die Kinder der Lilith von Isolde Kurz (1925) und weiteren Texten (Zingsem). Dieser vielgestaltig projizierten Lilith wurde 1996 eine Ausstellung Mythos Lilith von Regina H. Schmid im Frauenmuseum Bonn gewidmet. Es gibt nur eine einzige Abbildung von Lilith, nämlich das Terrakottarelief aus dem 2. Jahrtausend v. Chr. (wobei diese Identifizierung inschriftlich nicht bezeugt ist). Sie wurde als Umwerterin aller Werte gewürdigt, die - wie Nietzsche mit seiner Gott-ist-tot-Proklamation - die damals herrschende Ordnung zum Purzeln brachte und dafür durch das Gott-Adam-Gespann mit Verbannung regelrecht bestraft wurde. In der Rockoper Lilith von Walter Weyers fungiert sie als Hauptfigur (2003), die nach der kläglichen Zurückweisung einer gleichberechtigten Partnerschaft durch Adam gemeinsame Sache mit dessen zweiter Frau (und Göttin) Eva macht, um aus der archetypischen "Wiederholung der Vergeblichkeit der Liebe« heraus zu kommen. Der Psychiater Hans-Joachim Maaz hat unter dem >Lilithkomplex die Nachtseite des Weiblichen und Mütterlichen abgehandelt (2001/2003) und hat sich Lob und ebenso Kritik für seine These von der "neuen Mütterlichkeit» im Gefolge Liliths zugezogen, weil er zu wenig seinen eigenen Hinweis beachtet habe, dass der Lilith-Mythos ein typisch patriarchales, männliches Gewächs und Gespinst sei. Die Theologin Vera Zingsem hat Liliths weit verbreitete Spuren durch die Kulturen verfolgt bis hin zu Lilith als Symbolfigur der Frauenbewegung (2003). Neuerdings tritt Lilith in der Fantasyliteratur und in mytho-dramatischen Rollenspielen als Monster und Vampir auf, wogegen andere Lilith lieber in Gestalt der erosträchtigen Aphrodite aufleben lassen. In der Bibel kommt Lilith nur in Jesaja 34,14 vor als Dämonin, die in der Wüste zur Ruhe kommt. Luther hat bei seinem Übersetzen des sogenannten Alten Testamentes, der jüdischen Bibel, Lilith faktisch herausgestrichen, vielleicht aus Vorsicht und Angst auf dem Weg, einem gnädigen Gott $\mathrm{zu}$ begegnen. Auf manches wäre noch zu verweisen an Sekundärem und Hinzuerzähltem von der schillernden Lilith. Was lässt sich `Biographisches ausmachen?

(2.) Die Lilith-Mythologie: Gottes erster Versuch der Erschaffung einer Frau für den ersten Menschen Adam. Gott erschuf den ersten Menschen Adam und bemerkte nach kurzer Zeit einen Mangel oder sogar Defekt in seinem Schöpfungsprojekt: »Es ist nicht gut, dass der Mensch allein sei.« Und er schuf Lilith aus der gleichen Erde wie 
den Adam. Das ging nicht lange gut, und bald begannen die beiden, sich in endlose Streitereien zu verwickeln: »Ich will nicht unter dir liegen in >Missionarsstellung zur Erschaffung unserer Kinder«! »Ich will auf dir liegen, weil du die Unterlegene bist und ich der Überlegene, auch der Erste bin«!, versucht Adam sein Ersterschaffensein schamlos in Vorrechte umzumünzen. »Wir sind beide gleich, weil wir aus der gleichen Erde gemacht sind«!, kontert Lilith im Blick auf ihre gemeinsame Herkunft aus der Erdmutter. Und so redeten sie aufeinander ein und aneinander vorbei, der eine zur Sicherung seiner (angeblichen) Vorrangstellung und damit gegebenen Macht, die andere zum Zusammenleben auf gleicher Augenhöhe, in Gleichberechtigung. Bis Lilith den magischen Namen Gottes ausrief und sich in die Lüfte erhob, woraufAdam seinen Schöpfer-Gott mit tadelnder bis anklagender Stimme frontal und ohne eigenes Schuldbewusstsein und Scham anrief: »Gott der Welt, die Frau, die du mir gabst, ist weggelaufen und hat mich verlassen!" Dies war dem Weltenschöpfer peinlich und beschämend, so dass er schleunigst drei Engel der Lilith hinterherschickte, dass sie diese zurückholen sollten. Zu Adam sprach er: "Wenn sie zurückkehren will, gut. Wenn nicht, muss sie es auf sich nehmen, dass tagtäglich hundert ihrer Söhne sterben müssen." Lilith kehrte - zur Beschämung Gottes und des Adam - nicht zurück in die hierarchische Gott-Adam-Schöpfungsordnung, deren patriarchale Grundstruktur sie mit diesem Selbstbefreiungsschlag entlarvte (und unsterblich blieb, weil sie sich vor dem Sündenfall von Adam getrennt hatte, der seinerseits durch den Sündenfall mit Eva sterblich wurde). Auf diese Peinlichkeit reagierten der in die Klemme geratene Schöpfer-Gott und der männlich-narzistische Adam wiederum peinlich: Es ist nicht gut für Adam, dass er allein ist. Dies ist ein Mangel im Schöpfungsgefüge: Schöpfung geschieht unvorausdenklich als Auseinandertreten von Leben und Tod, von Nichts und Welt, als Ereignis der Differenz. Der einsame Adam ist als einziger, alleiniger Mensch eigentlich (noch) kein Mensch. Also muss Gott einen erneuten Erschaffungsversuch einer passenden Frau für Adam unternehmen und eine Frau erfinden und ins Leben rufen, die sich mit Adam zusammen in der Schöpfungswelt zurecht findet - und seine Männerphantasien erfüllt?

(3.) Die erste Eva (Proto-Eva): Gottes zweiter Versuch. Dieses Mal nahm Gott statt radamischer Erde< Knochen, Gewebe, Muskeln, Sehnen, Hornhaut, Blut zur Erschaffung der Frau, baute aus diesen Materialien ein Gerüst, überzog sie mit einer Haut, setzte Haarbüschel ein - aber Adam fand gar kein Gefallen an dieser Eva und Gott ließ, als er seinen Fehlschlag erkannte, die »erste chawah" auf 
Nimmerwiedersehen verschwinden. Diese Hawwa oder Hebe war die >Mutter allen Lebens`, die Erdgöttin von Jerusalem im Obstgartenparadies. Leben brachte sie mit der phallischen Schlange hervor. Später bekam sie einen Heros-Geliebten, nämlich Abdiheba, den Adam, und sie feierten jährlich die Heilige Hochzeit, nachdem Eva dem Adam den Liebes- und Todesapfel übergeben hatte. Und sie tötete ihn, um ihm ewige Jugend und Leben zu schenken - ein Mythos, der sich bis in die christologischen Jesus-Mythen um Tod und Auferstehung durchgehalten hat. »Den Namen nahm der jüdische Gott Jehova (Jehva = Eva) in Besitz und mit dem Namen ihr Paradies«, kommentiert Heide Göttner-Abendroth.

(4.) Gottes dritter Versuch: Die >Frau aus der Rippe Adams. Gott ließ nach dem erneuten peinlichen Rückschlag nicht locker, um Adam eine genehme, passende 'Gehilfin Gott über seinen Fehlschlag gegenüber Adam geschämt hat oder ob sich Gott gar nicht schämen kann, gibt der Mythos nicht zu erkennen.) Und so kam es zum dritten Versuch, nämlich die `Frau aus einer Abspaltung von Adam zu gestalten: aus Adams Rippe, wie der Jahwist in 1. Mose 2 erzählt. Mit dieser Mythologie werden die göttliche und menschliche Männermacht und der Gebärneid der Männer legitimiert, stellt feministische Theologie fest, denn: Genau genommen wird diese Eva gar nicht erschaffen, sondern Eva kommt als eine >Abspaltung von Adam hinzu. So ist "die größte Gewalttat der Religionsgeschichte nach wie vor nicht nur unbenannt, sondern auch unbewußt geblieben" (Zingsem).

Mit dem schamlosen und beinahe komplett gelungenen Versuch der Patriarchalisierung der israelitisch-jüdisch-christlichen Schöpfungsmythologie, durch den aus ursprünglicher Eva-Mythologie eine Adam-Mythologie geworden ist, stehe die Erschaffung der Frau (wieder) noch aus, zumal die zweite Eva ja nicht die Frau Adams ist, sondern sie ist - wie bereits angesprochen - die Tochter Adams. Gott nahm nämlich, wie in 1. Mose 2,19-23 erzählt wird, eine Rippe aus Adams Brust, während dieser von Gott in einen Tiefschlaf geschickt wurde, und gestaltete daraus Eva. Als Adam aufwachte, war er begeistert von dieser Frau. Und von beiden heißt es: Sie waren nackt und schämten sich nicht (1. Mose 2,25). Die im dritten Versuch erschaffene zweite Eva ist `Fleisch von Adams Fleisch`, also seine Tochter: "Nicht Ödipus steht an der Wiege unserer Beziehungskultur, sondern ein Vater, der sein Begehren auf die Tochter richtet « (Zingsem), wobei der Inzest in der Götterwelt keine moralischpeinliche Schranke und Scham-Grenze darstellte und zumal es sich 
hier um reine Männer-Geschichten (-Mythen) handelt ohne Blick auf wirkliche Frau(en).

\section{Machtkampf Nr. 2: Der `Fallı ins Menschsein: Strafe und Freiheitszuspruch?}

Adam und Eva schämten sich nicht. Sie leben ohne die Fremdheit des Anderen und seiner/ihrer selbst wahrzunehmen, ohne Differenzund Kontrasterfahrung. Sie sind noch ganz bei sich selbst und ihrer Welt zu Hause, ohne Scham und Schuld, also imVergleich zu uns >Gefallenen so etwas wie Vor-Menschen. Ihre Wahrnehmung und ihre Welt atmen eine einzige Ordnung und sind nicht gespalten in Vertrautes und Fremdes, in Ordnungen, die Entscheidungen verlangen. Im Fremden hingegen »bin ich außer mir und außerhalb der jeweils bestehenden Ordnungen [...]. Ein solcher Ausbruch und Einbruch des Fremden (sc. wie im Sündenfall) setzt allerdings jene Ordnungen voraus, die er durchbricht«, nämlich die Paradies-Ordnung (Bernhard Waldenfels). Diese identisch-paradiesische Grundstruktur des Menschenpaares und seiner Welt weist auf eine Art Urzustand zurück, den sich Menschen bis heute als Heilsort der Identität und der Konvergenz in einem vor- und übergeordneten Dritten phantasieren. Mit dem `Sündenfall, mit dem Schämen und der Erfahrung der Schuld, wird dem Menschen eingeschrieben, »dass er, wo immer er lebt, ein Differenzwesen ist und dass er mit profanen oder religiösen Praktiken versucht, diese Differenz zu überwinden, um sich oder die Welt ganz zu machen" (Peter Gross). Was wäre gewonnen und was wäre an Menschlichkeit verloren, wenn wir ohne Schamwiderfahrnisse und ohne Schamerfahrungen lebten? Diese Frage zielt auf den Unterschied zwischen 1. Mose 2,25: „Und obwohl die beiden nichts anhatten, der Mensch als Mann und seine Frau, schämten sie sich nicht", und 1. Mose 3,7: "Da wurden beiden die Augen geöffnet und sie erkannten, dass sie nichts anhatten « (zitiert nach der Bibel in gerechter Sprache). Für diesen Vergleich müssen wir bei der jahwistischen Schöpfungsmythologie einsetzen: Im Paradies-Garten wuchs in der Mitte der (in nahezu allen Religionen bekannte) Baum des Lebens und dazu der Baum der Erkenntnis von gut und böse (1. Mose 2,4b-25). Vom Lebensbaum zu essen, war verboten (wohl durch Gott Jahwe):»Esset nicht davon. Rühret sie auch nicht an, dass ihr nicht sterbet." (1. Mose 3,3) Und dann nimmt das Drama seinen Lauf: Eva lässt sich von der Schlange, die in jüdischer Tradition als männlicherVerführer und in christlicher Überlieferung erstmals im Mittelalter mit einem Frauenkopf dargestellt wird, dazu überreden, 
von der verbotenen Frucht zu essen, was auch als Euphemismus für geschlechtlichen Verkehr zwischen Eva und der Schlange oder zwischen Eva und ihrem Mann gedeutet werden kann. Und mit diesem Biss in den Apfel erhält Eva dämonische Macht und eine sexuelle Bewusstheit, um ihren Mann zu verführen (wie sie Lilith als Dämonin zugeschrieben wurde). Die Frau hat mit der Übertretung des Verbotes Gottes Schuldbewusstsein und Scham in die Welt, in das Zusammenleben gebracht. Für dieses ihr Vergehen werden sie und Adam von Gott bestraft, schlussendlich mit der Vertreibung aus dem Wohnbereich Gottes. Die und der Schamlose ist übergriffig; er akzeptiert die Differenz nicht, die sich z.B. im Verbot Gottes manifestiert; er tastet den Anderen an und achtet ihn nicht in seiner Intimität und Einzigartigkeit.

Seit Augustin gibt es eine andere Version der Sündenfallsgeschichte:Adam habe aus Liebe zu Eva und nicht aus Stolz Gott gegenüber gesündigt. Adam wollte Eva nicht kränken und aß ebenfalls vom Lebens-, Liebes- und Todesapfel. Also: Eva bringt auf Grund ihrer weiblichen Schwäche, nämlich sowohl wegen ihres Stolzes als auch ihres Ungehorsams gegen den Willen Gottes, Schuld und Scham in die Welt, Adam hingegen handelte männlich-tugendhaft aus Liebe und ist somit entschuldigt - was zur Legitimierung des (auch religiösen, in diesem Fall christlichen) Patriarchates zu demonstrieren war. Es gibt seit der Aufklärung die weiter gehende Deutung des Sündenfalls als einer in Wirklichkeit revolutionären Befreiungsgeschichte aus dem über- oder vormenschlichen Paradies in ein echt menschliches Leben, in ein sterbliches, ambivalentes Menschsein mit Schuld, Scham und Tod, mit Liebe, Vertrauen und Hoffnungen. Es wird in 1. Mose 3 nicht von einem `Fall erzählt, sondern im Gegenteil »von einer Erhebung, einem Übergang von der Kindheit zum Erwachsenwerden, von glücklicher Unwissenheit zu gefährlichem, aber reifem menschlichem Wissen, vom animalischen Instinkt zu menschlicher Vernunft" (so z.B. Schiller: Etwas über die erste Menschengesellschaft nach dem Leitfaden der mosaischen Urkunde, 1789). Ist entsprechend die Verbannung aus dem Garten Eden »ohne Widerspruch die glücklichste und größte Begebenheit in der Menschengeschichte" (Schiller), also ein Segen und kein Fluch, wie schon etliche Gnostiker und z.B. Maimonides (1135-1204) vorschlugen? Es geht um ein Christentum, das »den Makel in ein Heilszeichen umdeutet und eine immerwährende Passion ohne endgültige Erlösung feiert" (Peter Gross). "War der Stachel (sc. aus 1. Mose 3) ein Schlüssel, der Adam und Eva zur Flucht verhalf? Ist nicht das grandiose Schauspiel der Kulturen und ihrer pausenlosen Überar- 
beitung letztlich eine Folge der Blöße, welche die ersten Menschen der biblischen Geschichte an sich entdeckt haben?« Blöße, deren Dynamik gerade entzogen bleibt und die sich in ambivalenten Bedürfnisbefriedigungen manifestiert? Dann könnte der nächste Schritt sein: „Gäbe es eine gelassene Religiosität, welche eine Transzendenz akzeptiert, ohne in diese eingehen zu wollen?« (Gross). Gut und böse unterscheiden zu müssen, ohne dies endgültig zu können, wäre dann nicht nur Strafe, sondern sowohl "heilsam als auch schädlich" (wie Julius Wellhausen, der Initiator der Genesis-Kritik, formulierte). Aus den bestraften Paradiesvertriebenen werden Paradiesflüchtlinge, aus dem Paradies wird die Natur, mit der der Mensch im Widerstreit liegt (Erich Fromm). Aber wie üblich in der Menschheitsgeschichte wurden auch hier die aufklärerische Emanzipation der Vernunft, etwa bei Schiller, durch übereifrige >Rationalisten die Befreiung von Schuld durch die `Religiösen vergegenwärtigt statt sich mit dem Paradox im Sinne Wellhausens zu >begnügen . Menschliches Leben ist mit dem Sündenfall ambivalent geworden, wie sich am Schämen zeigt, über das wir nicht Herr sind, weder über seinen Anfang noch über sein Ende.

Hier soll noch ein kurzer Seitenblick auf psychologisch orientierte Interpretationen der Sündenfalls-Mythologie geworfen werden. Carl G. Jung, sein Schüler Erich Neumann und auch z.B. Erich Fromm sahen in Eva den Archetyp der Einen Großen Mutter, die die Gegensätze von Himmel und Erde (und Unterwelt), von Erbarmen und Rächen, von Fürsorge und Zerstörung in sich schließt. Sigmund Freud vertrat mit Otto Rank die Theorie, dass Adam von Eva als der Muttergöttin erschaffen worden sei, dass Adam Eva verführt habe, dass Gott in der Gestalt einer Schlange und nicht Satan mit Eva geredet habe, dass also der Jahwist-Erzähler als Analysand ungewollt diese Mythologie auf den Kopf gestellt habe. Entsprechend kann der Sündenfall z.B. bei Freud nur negativ gesehen werden als Hemmnis für den Sexualtrieb. So gehe es in 1. Mose 3 um Mutterinzest, um Bewusstwerdung und Bestrafung, um geschlechtliche Zeugung (und die entsprechende Bestrafung mit sexuellem Verlangen und mit schmerzhaftem Gebären) und um den Beginn menschlicher Kultur (und die entsprechende Bestrafung mit Familienfürsorge und mühsamem Ackerbau): "An die Stelle der ursprünglichen, vorindividuellen Harmonie zwischen Mensch und Natur und zwischen Mann und Frau trat der Konflikt und der Kampf." Aber: "Der Mensch möchte seiner neu erworbenen Freiheit wieder entrissen werden und eben das Bewußtsein seiner selbst, das ihn zum Menschen macht, wieder verlieren." (Erich Fromm) Dieses Paradox ist dem 
sündigen Menschsein eingeschrieben und äußert sich im Schämen als Widerfahrnis und zugleich als Antworten.

\section{Was wir sonst noch von der Scham entdecken können}

Es könnte bisweilen das Missverständnis aufgekommen sein, als ob Scham (seit dem `Sündenfalls) eine zeitlose Eigenschaft von uns Menschen sei, etwa ein vererbter Makel (als sogenannte Erbsünde), eine humanbiologische, genetische Ausstattung und Disposition, so etwas wie ein Existential oder eine transzendentale Kategorie. Scham ist als eine bestimmte Beziehungsqualität zu verstehen, und sie ist in ihren Äußerungen gesellschaftlich, kulturell, religiös geprägt. Anders als Peinlichkeiten, die an der Oberfläche bleiben und repariert und verziehen werden können, bringen wir Scham nicht selbst hervor, sondern Scham widerfährt uns, besetzt uns, überwältigt uns im Modus des Begehrens in seinen verschiedenen Äußerungsweisen, Bedürfnissen, Befriedigungen. Scham verkleidet sich in ein uns zustoßendes Geschehen, das vorher war und unaufhellbar bleibt. Sie widerfährt in der Unerreichbarkeit des Selbst und des Anderen. Emmanuel Levinas meint: "In meiner Scham ist der Andere begehrt." Solche Scham hat nicht den Modus des Bewusstseins und begreifenden Vergegenwärtigens, sondern sie bleibt außerhalb von mir und befreit und zwingt mich zum eigenen Antworten. Das Subjekt der Scham ist außerhalb von mir, es überfällt mich. In der Scham, so fährt Levinas fort, bin ich nicht ein unschuldiger Antwortender, sondern "Usurpator und Mörder". Dies mag überzogen klingen, aber Levinas weist auf das Geschehen hin, dass ich im Scham-Widerfahrnis schon immer meine eigene Haut zu retten unternehme und mich damit in meiner Unfertigkeit und Dezentriertheit verfehle und dabei den Anderen stöter. Im Antworten nehme ich meine vom Anderen mir zustoßende Freiheit zurück, da es reiner Freiheit im Sinne der Paradies-Erzählung menschlich nicht gibt. »Indem sich die Freiheit im Bewußtsein der Scham entdeckt, verbirgt sie sich in der Scham selbst.« (Levinas) Theologisch bei Dietrich Bonhoeffer:

"Die Scham des Menschen ist der widerwillige Hinweis auf die Offenbarung, auf die Grenze, auf den anderen, auf Gott. Darum ist die Bewahrung der Scham in der gefallenen Welt die einzige - wenn auch höchst widerspruchsvolle - Möglichkeit des Hinweises auf die ursprüngliche Nacktheit und der Heiligung dieser Nacktheit; nicht weil die Scham an sich etwas Gutes wäre - das 
ist die moralistisch puritanische (sc. auch pädagogisiert bestrafende), ganz unbiblische Auffassung -, sondern weil sie widerwillig Zeugnis geben muß vom eigenen Abgefallensein."

Menschsein ist nicht `Existenz für sich`, was ein Leben ohne Scham und Schuld wäre, sondern ist dessen Infragestellung und Beschämung im Abfallen vom Anderen (Menschen, Gott). So wird in 1. Mose 3 m.E. weder ein Erbsünden-Dogma erzählt noch ein zu perfektionierender Emanzipations- und Erziehungsprozess des Menschengeschlechts zurück in ein schamfreies Aufgeklärtsein initiiert, sondern Menschsein als Paradox auf Messers Schneide wahrgenommen: Die vom Anderen geschenkte und aufgezwungene Freiheit empfindet die Freiheit als Schämen, weil sie entdeckt, dass sie in ihrer Ausübung »usurpatorisch« ist, und dass sie vom Anderen gerettet werden muss, um nicht verloren zu gehen in Bedürfnisbefriedigungen. Freiheit kann sich nicht ganz nackt, unschuldig, ‘für sich`, ohne Scham manifestieren, wie sich einst im Paradies Adam und Eva (und vorher Lilith) gegenseitig >rein und lauter wahrgenommen haben und wie Gottes symbolische Bekleidungsaktion diese menschliche, ambivalente, paradoxe Freiheit auf den Weg gebracht hat.

\section{Zum Schluss}

Scham wird in unserer Selbstermächtigungsgesellschaft radikal individualisiert und dadurch immer mehr zu einer Schwäche degradiert, überspielt, ausgelöscht, weil die sich darin ereignende Kontrasterfahrung mit dem Fremden des Anderen und meiner selbst als elementare Störung zum Verschwinden gebracht werden soll. Scham wird zur Mangel-Erfahrung herabgewürdigt, dessen Geschenkcharakter durch therapeutische Maßnahmen aller Art ausgetrieben werden soll. Scham hält eine nicht festlegbare Lücke offen, durch die man verbunden wird, wenn sie in >Freud und Leid In dem Maße wie heute vor allem medial eingeschärfte Identität, Gleichheit und Alternativlosigkeit immer penetranter als Ziel unserer Gesellschaft fungieren und Differenz und Einzigartigkeit verleugnet werden, wird unsere Gesellschaft oberflächlich, sschamlos`, sozial-autistisch. Wir verdrängen, dass wir den Anderen und uns selbst schon immer verfehlen, was etwas anderes ist als moralische, rechtliche Verfehlungen. >In, mit und unter diesem mit Scham getränkten Schon-immer-Verfehlen ereignen sich Widerfahrnisse, die uns befreien, verändern, Verantwortung auftragen, zum Antworten ermächtigen, die uns lernen, vertrauen, lieben lassen und die uns zugleich usurpieren, hassen, sogar töten lassen. Mit dem Wort ıScham 
ist auf das Paradoxe menschlichen Zusammenlebens und Lebens hingewiesen.

- Uwe Gerber, Studium der Evangelischen Theologie und Philosophie, 1964 Promotion, 1968 Habilitation in Basel. Akademischer Oberrat für Theologie und Sozialethik an der TU Darmstadt und außerordentlicher Professor für Systematische Theologie der Universität Basel. 